\title{
SARS-CoV-2 infection inducing severe flare up of Deficiency of Interleukin Thirty-six (IL-36) Receptor Antagonist (DITRA) resulting from a mutation invalidating the activating cleavage site of the IL-36 receptor antagonist
}

\author{
Alizée Bozonnat ${ }^{1,2} \cdot$ Florence Assan $^{3} \cdot$ Jérôme LeGoff ${ }^{4} \cdot$ Emmanuelle Bourrat $^{1,2} \cdot$ Hervé Bachelez $^{3,5}$
}

Received: 27 April 2021 / Accepted: 24 May 2021 / Published online: 26 June 2021

(c) The Author(s), under exclusive licence to Springer Science+Business Media, LLC, part of Springer Nature 2021

To the Editor,

The Deficiency of Interleukin Thirty-six Receptor Antagonist (IL36RA) (DITRA) is a rare autoinflammatory monogenic syndrome resulting from the interaction of autosomalrecessive or double composite heterozygous mutations of $I L 36 R N$, the gene encoding IL-36RA, with environmental triggers such as infection and pregnancy. This sporadic or familial syndrome is characterized by recurrent acute flares of generalized pustular psoriasis (GPP, OMIM 614,204), with life-threatening potential. Recently, we reported for the first time documented viral respiratory tract infections (RTI) as potent triggers in acutely flaring psoriasis of different subtypes, including GPP [1]. We herein report a case of acute, severe DITRA flare triggered by SARS-CoV-2 infection, and discuss the implications regarding disease pathogenesis and management in view of immunologic profiling studies.

The causal mutation in this pedigree has already been reported as a p.V2F aminoacid substitution in the IL-36RA protein resulting from a homozygous c. $4 \mathrm{G}>\mathrm{T}$ nucleotidic variant of IL36RN in 3 out of 4 siblings born from related

Hervé Bachelez

herve.bachelez@aphp.fr

1 Department of General Pediatrics, Infectious Disease and Internal Medicine, Hôpital Robert Debré, AP-HP, Paris, France

2 Department of Dermatology, Reference Center for Genodermatoses and Rare Skin Diseases (MAGEC-Saint Louis, ), Hôpital Saint-Louis, AP-HP, Paris, France

3 Laboratory of Genetics of Skin Diseases, INSERM U1163, Imagine Institute, Université de Paris, Paris, France

4 Department of Virology, Hôpital Saint-Louis, AP-HP, Université de Paris, Paris, France

5 Department of Dermatology, Hôpital Saint-Louis, AP-HP, Paris, France parents of Algerian descent [2]. This mutation is unique in impairing the cleavage of the immature form of IL-36RA at aminoacid residue 2 by proteases, mainly by neutrophilderived elastase, a mandatory step for IL36RA optimal biological activity [3]. There was no mutation of CARD14 or $A P 1 S 3$ in any individual from the pedigree, and the parents and one sibling have been shown to be heterozygous carriers of the IL36RN c. $4 \mathrm{G}>\mathrm{T}$ mutation, and were free of any skin or mucosal lesions (see Fig. 1 for the pedigree).

A 16-year-old male patient (Fig. 1) suffering from early onset DITRA with frequently relapsing typical GPP with systemic symptoms and a geographic tongue, had been in complete stable clinical remission for several months after withdrawal from adalimumab treatment, which had been initiated following failures of acitretin and cyclosporine. In late september 2020 , he reported a rapidly disseminating pustular eruption with superficial scaling and yellow crusts accompanied with asthenia and myalgia, occurring 2 days after the onset of the first COVID-19 symptoms consisting in cephalalgia, fever, pharyngitis with anosmia and ageusia. Three weeks later, he was clinically assessed for a GPP/ DITRA eruption covering $60 \%$ of the body surface area. The 5 points scale Generalized Pustular Psoriasis Physician Global Assessment (GPPPGA) score performed by a trained physician (HB) was 3 (moderate) with presence of pustular lakes, while the generalized pustular psoriasis area and severity index (GPPASI) was 45,2 for a maximal value of 72 [4]. (For full details, see online additional data supplementary ESM1). Biological investigations revealed peripheral blood leukocytosis with neutrophilia (neutrophil count: $17,610 / \mathrm{mm}^{3}$ and lymphocyte count: $2460 / \mathrm{mm}^{3}$ ), while C-reactive protein (CRP) serum level was normal. Polymerase chain reaction (PCR)-based analysis of nasopharyngeal swabs, using a Filmarray multiplex test detecting sixteen respiratory viruses and four bacteria (Chlamydophila 


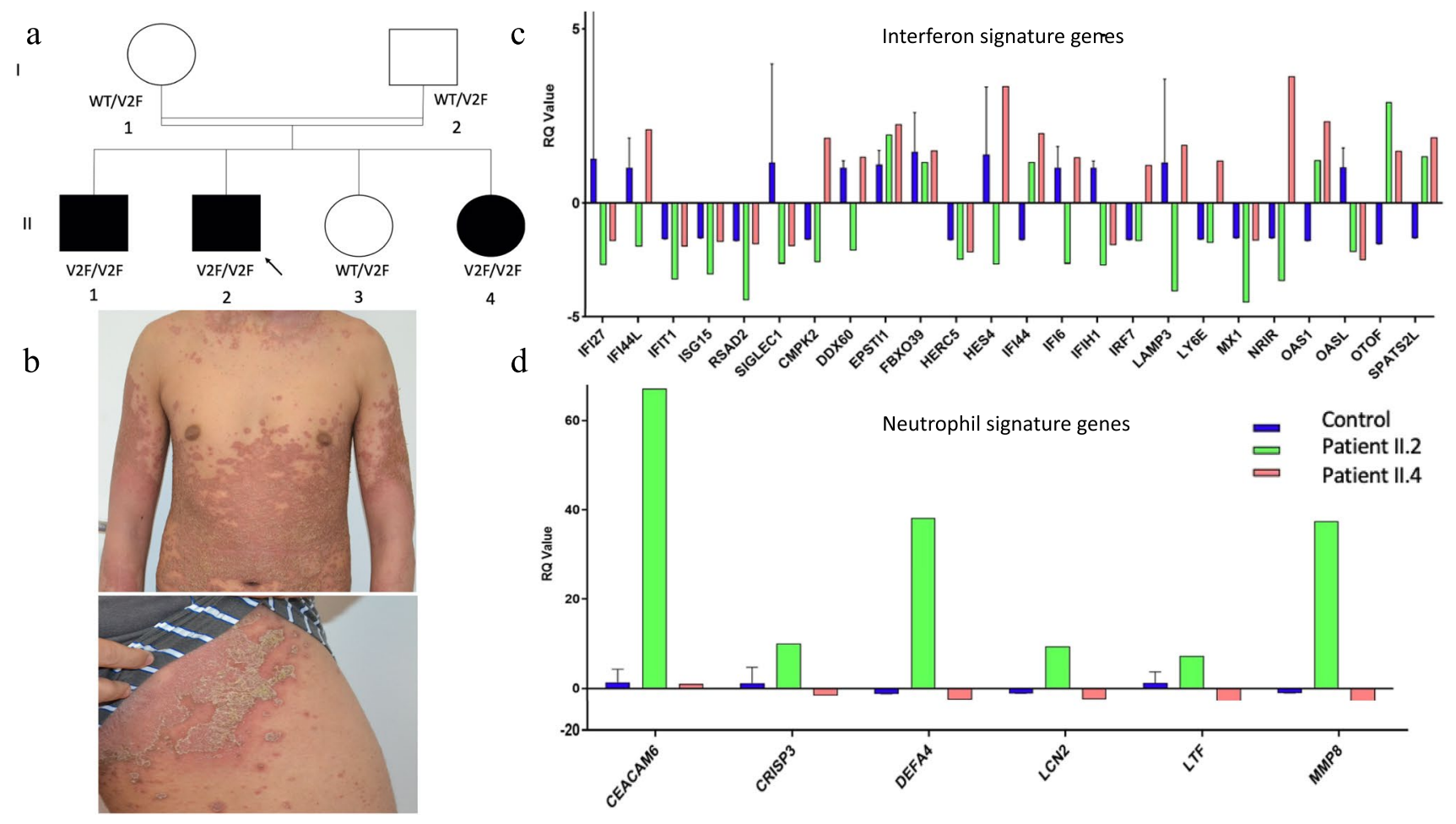

Fig. 1 On the left, a Family pedigree. b Acute flare up of generalized pustular psoriasis of the proband (patient II.2). On the right, Interferon Signature Genes (ISG) and neutrophil signature genes mRNA quantitative assessment in whole blood from patient II.2 (proband) and patient II.4 (proband's sister). c Median fold expression of interferon-stimulated genes (ISG)*. d Median fold expression of neutrophil signature genes**. *ISG signature assessment: Total RNA was extracted from whole blood using the PAXgene RNA isolation kit (PreAnalytix, Hombrechtikon, Switzerland). The ISG mRNA expression was assessed using the nanoString (NanoString, Seattle, WA, USA) procedure; $200 \mathrm{ng}$ of RNA was hybridized to the probes (a reporter probe and a capture probe) at $67{ }^{\circ} \mathrm{C}$ for $16-21 \mathrm{~h}$. Samples

pneumonia, Mycoplasma pneumonia, Legionella pneumophila, Bordetella sp.) (Biomérieux, Macry-l'Etoile, France) confirmed the diagnosis of COVID-19 with the exclusive presence of SARS-CoV-2. Both $\mathrm{E}$ and $\mathrm{N}$ genes were detected with Cycle Threshold value at 38 (Xpert ${ }^{\circledR}$ Xpress SARS-CoV-2, Cepheid, Sunnyvale, CA). The skin rash partially resolved under topical steroid treatment with betamethasone once daily, while the SARS-CoV-2 PCRbased detection in nasopharyngeal swabs became negative one week later. The serological detection of anti-SARSCoV-2 nucleocapsid $(\mathrm{N})$ and spike (S) IgG antibodies using a chemiluminescent microparticle immunoassay (Architect, Abbott, Sligo, Ireland), performed 32 days after COVID-19 symptoms onset, was strongly positive (index 5,79 ), whereas PCR and serological detection of SARS-CoV-2 were negative in his heterozygous carrier mother (Fig. 1 patient I.1), in his 5-year-old sister (patient II.4) with mild and limited GPP/DITRA skin lesions (GPPPGA:2; GPPASI:3), as well were then inserted into the nCounter Prep Station for the removal of excessive probes, purification, and immobilization onto the internal surface of a sample cartridge for 2-3 h. Finally, the sample cartridge was transferred to the nCounter Digital Analyzer, where color codes were counted and tabulated for each target molecule. Count numbers obtained for the ISGs were normalized by the geometric mean of three housekeeping genes count numbers ( $\beta$-actin, HPRT1 [hypoxanthine phosphoribosyltransferase 1] and POLR2A [RNA polymerase II subunit A]) as well as the negative and positive control values using the nSolver software. The median fold change of the ISGs compared to the median of the healthy controls. $* *$ Neutrophil signature genes include CEACAM6, CRISP3, DEFA4, LCN2, LTF, and MMP8 as in his 18 year-old brother (patient II.1) who showed plicaturated tongue as the only DITRA symptom since birth, both homozygously mutated for IL36RN. The SARS-CoV2-induced GPP flare completely resolved within 3 months after restart of adalimumab treatment, without relapse over a 6 months follow-up period. To note, the patient's medical records revealed one episode of GPP/DITRA acute flare already triggered by infection with Bordetella pertussis.

As GPP has been associated with both neutrophilic and various degrees of type I interferon (IFN-I) transcriptomic signatures [5], IFN signature genes (ISG) and neutrophil signature genes (NSG) mRNA expression was quantitated using whole blood nanostring technology as previously described [6], at day 32 post-onset of COVID-19 symptoms in the proband and in his mildly affected sister. Results showed a strongly increased neutrophilic signature in the proband, but not in the mildly affected sister, while mRNA expression of ISG was in the normal range in both cases (Fig. 1). 
To our knowledge, this is the first case of DITRA flare-up induced by SARS-CoV-2 infection. Indeed, the complete, stable remission status in patient 1 several months before the COVID-19 infection onset, the personal prior history of post-infectious DITRA flare-up, and previous reports of GPP flares occurring concomitantly or immediately after RTI including a recent case of post-SARS-CoV-2 new onset acral PP without evidence of DITRA, all support the impact of infectious triggers, especially of viral RTI, on the evolutive profile of this autoinflammatory syndrome $[1,7]$. The molecular and cellular interactions linking RTI- and specifically SARS-CoV-2-induced bronchial inflammation, and skin and systemic inflammation in GPP, remain unknown to date. Although IFN-I, which has been showed to be instrumental in an effective anti-SARS-CoV-2 immune response [8] and to be upregulated in a subset of patients with GPP, might be involved in this inflammatory cascade, we did not find evidence for its deregulation, in discrepancy with previous reports in GPP without Covid19 [5]. This might be due either to the late sampling after the infection onset in our patient, or to alternative innate immune mechanisms operating in some patients. Likewise, the strong transcriptomic neutrophilic blood signature is in keeping with previous data in lesional skin of GPP patients showing upregulation of IL-36- and of neutrophil-attracting and/or activating chemokines such as CXCL-1, CXCL-2 and CXCL-8, among others [9]. Moreover, this case emphasizes the key role of elastase-driven catalytic cleavage of immature, biologically inactive form of IL-36RA for an optimal function of the antagonist, which is mandatory to prevent acute skin neutrophilic inflammation following environmental triggering events including SARS-CoV-2 infection [3]. Our observation has some limitations: first, the blood transcriptome assessment was performed in the proband 32 days after symptoms onset. Second, these experiments do not address the signatures in lesional skin or in lung tissues.

Finally, and due to the orphan natures of GPP and of DITRA, it is still unknown if inborn errors leading to upregulation of the IL-36 pathway and to GPP are protective or deleterious with regard to SARS-CoV-2 infection outcomes. In this respect, it is interesting to notice that COVID-19 infection was benign but symptomatic in our patient, despite the absence of upregulated IFN-I response. Given the yet equivocal prognostic value of a strong neutrophilic signature in COVID-19, it would be of interest to investigate the clinical outcomes and biological correlates of SARS-CoV-2 infection in neutrophilic systemic diseases such as GPP and DITRA on a larger scale, as currently only data in patients with psoriasis vulgaris are available at the population level. Finally, the growing evidence for a role of the IL-36 pathway in bronchial inflammation and the present case pave the way for mechanistic studies aiming to decipher the molecular and cellular links between RTI and psoriasis flare-ups.
Supplementary Information The online version contains supplementary material available at https://doi.org/10.1007/s10875-021-01076-6.

Acknowledgements We are grateful to Yanick Crow and Marie-Louise Frémond for technical and scientific support and discussion, Fadela Akroun and Mourad Djermane for their help in referring patients and Christian Thoma from Boehringer-Ingelheim for allowing us to use GPPASI and GPPPGA scoring. We also thank the patient and his family who accepted to report their history and participated in our study.

Author Contribution $\mathrm{AB}$ and $\mathrm{HB}$ wrote the manuscript. FA and $\mathrm{HB}$ performed the experiments and arranged the figures. $\mathrm{AB}$ and $\mathrm{EB}$ ongoing clinical care for the patient. HB supervised the study. All authors edited and approved the final version.

Funding This work and the laboratory of Genetics of Skin Diseases are supported by the French Ministry for Research (Programme Hospitalier de Recherche Clinique 2021, GENOPSO and GENPSO projects), the French Society for Dermatology (SFD), Amgen Inc., and by a restricted grant from Pfizer.

Data Availability All data and materials can be obtained by contacting the corresponding authors.

\section{Declarations}

Ethical Statements The study was approved by the Leeds (East) Research Ethics Committee (reference 10/H1307/132) and by the Comité de Protection des Personnes (ID-RCB/EUDRACT: 2014-A01017-40 and 2011-A00820-41). Written informed consent was obtained from all patients before inclusion.

Consent to Participate The patient and the patient's mother have consented to the submission of the case report to the journal.

Consent to Publish The patient and the patient's mother have consented to the submission of the case report to the journal.

Conflicts of Interest Alizée Bozonnat, Florence Assan, Jérôme Le Goff and Emmanuelle Bourrat declare no conflict of interests nor any competing interests. Hervé Bachelez had paid consulting activities for AbbVie, Almirall, Boehringer-Ingelheim, Janssen, Kyowa-Kirin, Leo Pharma, Novartis, UCB, and Pfizer.

\section{References}

1. Sbidian E, Madrange M, Viguier M, Salmona M, Duchatelet S, Hovnanian A, et al. Respiratory virus infection triggers acute psoriasis flares across different clinical subtypes and genetic backgrounds. Br J Dermatol. 2019;181(6):1304-6. https://doi.org/10. 1111/bjd.18203.

2. Bal E, Lim AC, Shen M, Douangpanya J, Madrange M, Gazah R, et al. Mutation in IL36RN impairs the processing and regulatory function of the interleukin-36-receptor antagonist and is associated with DITRA syndrome. Exp Dermatol. 2019;28(10):1114-7. https://doi.org/10.1111/exd.13387.

3. Macleod T, Doble R, McGonagle D, Wasson CW, Alase A, Stacey $\mathrm{M}$, et al. Neutrophil Elastase-mediated proteolysis activates the anti-inflammatory cytokine IL-36 Receptor antagonist. Sci Rep. 2016;6:24880. https://doi.org/10.1038/srep24880. 
4. Bachelez H, Choon SE, Marrakchi S, Burden AD, Tsai TF, Morita A, Turki H, Hall DB, Shear M, Baum P, Padula SJ, Thoma C. Inhibition of the Interleukin-36 Pathway for the Treatment of Generalized Pustular Psoriasis. N Engl J Med. 2019;380(10):981-3. https://doi.org/10.1056/NEJMc1811317 (PMID: 30855749).

5. Catapano M, Vergnano M, Romano M, Mahil SK, Choon SE, Burden AD, et al. IL-36 Promotes Systemic IFN-I Responses in Severe Forms of Psoriasis. J Invest Dermatol. 2020;140(4):816826.e3. https://doi.org/10.1016/j.jid.2019.08.444.

6. Lepelley A, Martin-Niclós MJ, Le Bihan M, Marsh JA, Uggenti C, Rice GI, et al. Mutations in COPA lead to abnormal trafficking of STING to the Golgi and interferon signaling. J Exp Med. 2020;217(11):e20200600. https://doi.org/10.1084/jem.20200600.

7. Mathieu RJ, Cobb CBC, Telang GH, Firoz EF. New-onset pustular psoriasis in the setting of severe acute respiratory syndrome coronavirus 2 infection causing coronavirus disease 2019. JAAD
Case Rep. 2020;6(12):1360-2. https://doi.org/10.1016/j.jdcr.2020. 10.013.

8. Zhang Q, Bastard P, Liu Z, Le Pen J, Moncada-Velez M, Chen $\mathrm{J}$, et al. Inborn errors of type I IFN immunity in patients with life-threatening COVID-19. Science. 2020;370(6515):eabd4570. https://doi.org/10.1126/science.abd4570.

9. Johnston A, Xing X, Wolterink L, Barnes DH, Yin Z, Reingold L, et al. IL-1 and IL-36 are dominant cytokines in generalized pustular psoriasis. J Allergy Clin Immunol. 2017;140(1):109-20. https://doi.org/10.1016/j.jaci.2016.08.056.

Publisher's note Springer Nature remains neutral with regard to jurisdictional claims in published maps and institutional affiliations. 